\title{
Intraoperative argon-plasma coagulation treatment for patients with malignant pleural mesothelioma
}

\author{
AKIRA IYODA $^{1}$, YOKO AZUMA ${ }^{1}$, TAKASHI SAKAI ${ }^{1}$, SATOSHI KOEZUKA $^{1}$, \\ HAJIME OTSUKA $^{1}$, NAOBUMI TOCHIGI ${ }^{2}$, KAZUTOSHI ISOBE ${ }^{3}$ and ATSUSHI SANO ${ }^{1}$ \\ ${ }^{1}$ Division of Chest Surgery, Department of Surgery, ${ }^{2}$ Department of Surgical Pathology, \\ ${ }^{3}$ Division of Respiratory Medicine, Toho University School of Medicine, Tokyo 143-8541, Japan
}

Received January 5, 2021; Accepted July 1, 2021

DOI: $10.3892 / \mathrm{mco} .2021 .2350$

\begin{abstract}
Malignant pleural mesothelioma (MPM) is often associated with asbestos exposure and carries an extremely poor prognosis. The present study assessed the effectiveness of argon plasma coagulation (APC) treatment in patients with MPM who underwent radical pleural decortication (PD). The clinical data from 11 patients who underwent radical PD treated with APC at Toho University Omori Medical Center from July 2015 to March 2020 were retrospectively analyzed. Clinical features, local recurrence, and clinical prognoses were evaluated. The median overall survival was 18.5 months, and the 1- and 2-year overall survival rates were 71.6 and $43.0 \%$, respectively. One patient survived 5 years but had recurrent tumors. The median disease-free survival was 11.1 months. The 1- and 2-year disease-free survival rates were 49.9 and $12.5 \%$, respectively. Three patients had no recurrences, two of whom were followed continuously (39.6 and 10.2 months). The present study revealed that APC treatment for MPM might be associated with good survival and prognosis. APC as an additional intraoperative treatment for patients with MPM may be further investigated with larger multi-center clinical trials to support its efficacy.
\end{abstract}

\section{Introduction}

Malignant pleural mesothelioma (MPM) is often associated with asbestos exposure and carries an extremely poor prognosis (1). The mean latency of MPM following asbestos exposure is 40 years (1). Although several non-surgical treatments for MPM are available, the role of surgery is still important (1). Extra-pleural pneumonectomy (EPP) has long been considered a radical approach; however, the Mesothelioma and Radical Surgery (MARS) study reported

Correspondence to: Dr Akira Iyoda, Division of Chest Surgery, Department of Surgery, Toho University School of Medicine, 6-11-1 Omori-Nishi, Ota-ku, Tokyo 143-8541, Japan

E-mail: aiyoda@med.toho-u.ac.jp

Key words: mesothelioma, argon plasma coagulation, decortication that EPP has a 14.4-month median survival and might harm patients (2). Thereafter extended pleural decortication (PD) was recommended over EPP due to fewer complications (1).

PD entails removal of the parietal and visceral pleura with sparing of the pulmonary parenchyma in an effort to excise all macroscopic tumors (3). Patients with PD have lower complication and mortality rates than patients with EPP; however, complete resection (R0) is extremely difficult with little improvement in prognosis (3). As additional intraoperative treatment, photodynamic therapy (PDT) can improve the prognosis of patients with MPM (4); however, PDT is currently not authorized for use in patients with MPM in Japan.

Argon plasma coagulation (APC) is effective for tumor resection or hemostasis with minimal complications and is commonly used for treatment of endobronchial tumors (5-7); however, there are no reports involving the use of APC in the treatment of MPM. We have mainly used APC for hemostasis during PD since 2015, and we recently noticed an anti-tumor effect for patients with MPM. The aim of this study was to determine the effectiveness of APC in the treatment of patients with MPM who undergo a radical PD.

\section{Materials and methods}

Ethics approval. This study was approved by the Ethics Committee of Toho University Omori Medical Center (M20085).

Study population. APC was performed for hemostasis after decortication on all patients who underwent a radical PD from July 2015 to March 2020. We retrospectively analyzed clinical data from all 11 patients who had undergone a radical PD with APC treatment at Toho University Hospital from July 2015 to March 2020. The clinical features, local recurrence, and prognoses were further evaluated.

The following parameters were retrieved from the medical records: Gender, age, pathologic diagnoses, pathologic TNM staging, adjuvant therapy, local recurrences, and outcomes.

Surgical methods. We performed a radical PD through the 5 and 9 th intercostal spaces. Of note, the 9 th intercostal space was used for resection of pleura at the costodiaphragmatic recess of the pleural cavity. All visceral and parietal pleura, 
including the interlobar spaces, were resected with caution to avoid the pericardium and peritoneum, thus minimizing the risk of tumor dissemination to the pericardial or abdominal cavities. After pleural resection, we performed APC to the chest wall while avoiding the nerves, vessels, and pericardium.

$A P C$. The patients received standard rather than novel or experimental clinical treatment. All patients were treated with an ERBE Argon Plasma Coagulator VIO300D/APC2 (Elektromedizin $\mathrm{GmbH}$ ). The argon gas flow rate was 2 1/min with a maximum electrical power setting of $80 \mathrm{~W}$. The APC applicator was $110 \mathrm{~mm}$ in length and $2.3 \mathrm{~mm}$ in diameter.

Statistical analysis. We evaluated the prognosis and survival time, based on Kaplan-Meier analysis, from the date of surgery until the time of recurrence or death or last follow-up evaluation, and univariate comparison was performed using the log-rank test. All statistical analyses were performed using JMP software, version 14.0 (SAS Institute Inc.).

\section{Results}

Characteristics of the study population. The patient characteristics are shown in Table I. Of the 11 patients, the oldest patient was 79 years of age. No complications were associated with APC. One patient had a sarcomatoid mesothelioma. One inpatient died due to a cardiac arrhythmia (Table II).

Prognoses. The median overall survival was 18.5 months. The 1- and 2-year overall survival rates were 71.6 and $43.0 \%$, respectively (Fig. 1). One patient survived 5 years, but had recurrent tumors. The median disease-free survival was 11.1 months. The 1- and 2-year disease-free survival rates were 49.9 and $12.5 \%$, respectively (Fig. 2). Three patients had no recurrences, two of whom had been followed continuously (39.6 and 10.2 months). On overall survival and disease-free survival, there was no significant difference between patients with epithelioid type and with non-epithelioid (overall survival, $\mathrm{P}=0.8558$, disease-free survival, $\mathrm{P}=0.2559)$.

\section{Discussion}

PD for patients with MPM is considered less invasive compared to EPP, and PD is recommended for some patients over EPP (1); however, PD alone is not sufficient to cure MPM because the goal of surgery in patients with MPM is to achieve macroscopic complete resection, while multimodal treatments, including additional intraoperative treatments for local control, have been attempted $(1,4)$. Friedberg et al (4) performed PDT therapy after radical pleurectomy with good outcomes and concluded that PDT might have a role in extending survival via direct cell death, breakdown of tumor neovasculature, and/or the immune response, although PDT does not appear to have a positive impact on local control (4). Unfortunately, PDT cannot be used for patients with MPM in Japan due to a lack of social insurance system coverage.

APC is considered a minimally invasive procedure with fewer complications compared to the Nd-YAG laser. APC is commonly used for treatment of endobronchial tumors given the benefits of minimal smoke and good visual control (7).
Table I. Clinical characteristics of patients with malignant pleural mesothelioma.

\begin{tabular}{lc}
\hline Clinical factor & Number of patients \\
\hline Patient number & 11 \\
Age, mean (range) & 65.8 (48-79) \\
Sex & 7 \\
Male & 4 \\
Female & \\
Location & 7 \\
Right side & 4 \\
Left side & \\
Pathological TNM stage & 7 \\
IA(T1N0M0) & 3 \\
IB(T2N0M0) & 1 \\
IIIA(T3N1M0) & \\
Pathological diagnosis & 5 \\
Epithelioid & 5 \\
Biphasic & 1 \\
Sarcomatoid & \\
\hline
\end{tabular}

Table II. Frequency of each complication-related factor.

\begin{tabular}{lc}
\hline Clinical factor & Total \\
\hline Adjuvant therapy & \\
Chemotherapy & 9 \\
No chemotherapy & 2 \\
Adjuvant chemotherapy & 4 \\
CDDP+PEM & 5 \\
CBDCA+PEM & \\
Recurrence & 7 \\
Positive & 3 \\
Negative & 1 \\
Inpatient death & \\
Recurrence in CDDP+PEM & 2 \\
Positive & 2 \\
Negative & \\
Recurrence in CBDCA+PEM & \\
Positive & 4 \\
Negative & 1 \\
\hline
\end{tabular}

APC is useful for intraoperative hemostasis without severe complications, and APC results for tumors may be equal to the reported laser results (7).

We have mainly used APC for hemostasis during PD since 2015 and we recently noticed an anti-tumor effect among patients with MPM. Although APC is useful for intraoperative hemostasis in patients with MPM, our study showed that APC may have an anti-tumor effect secondary to coagulation. In the present study 1 patient survived 5 years and 3 patients had disease-free survival with the median overall survival 


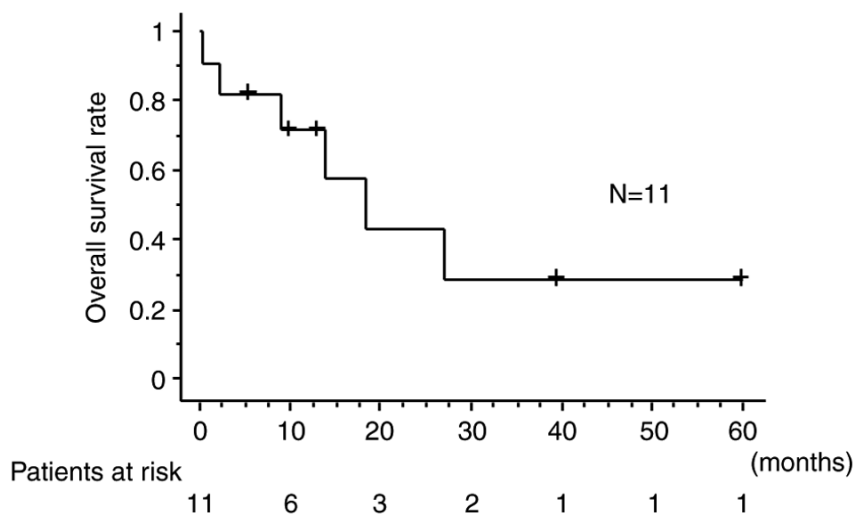

Figure 1. Overall survival curve of patients with malignant pleural mesothelioma.

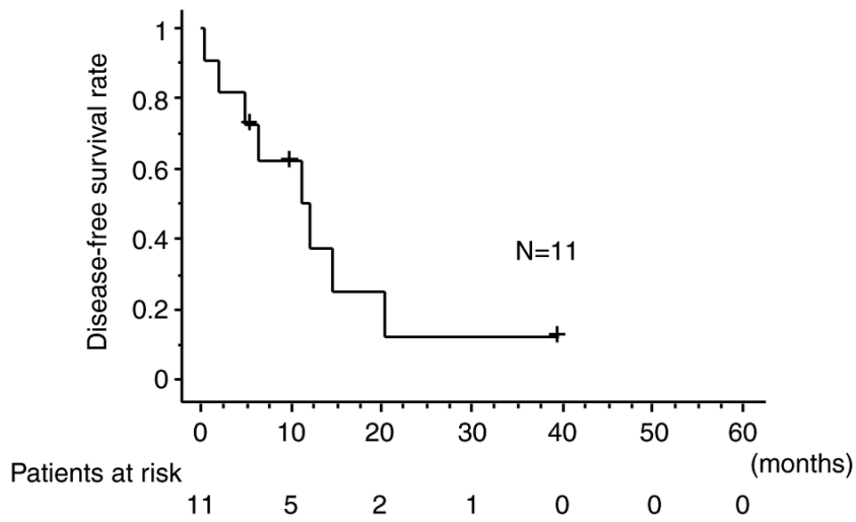

Figure 2. Disease-free survival curve of patients with malignant pleural mesothelioma.

of 18.5 months and the median disease-free survival of 11.1 months. A prior phase I trial of intraoperative hyperthermic treatments using cisplatin and gemcitabine for MPM revealed that the median overall and recurrence-free survival times were 20.3 and 10.7 months, respectively, the results of which were similar to our results (8), although chemotherapy of pemetrexed plus cisplatin for MPM revealed the median overall survival of 16.1 months (9). Our study revealed longer median survival compared to group with chemotherapy alone. Considering the rationale underlying PD is to achieve macroscopic complete resection without microscopic complete resection, this is the first report demonstrating the usefulness of APC for patients with MPM. Indeed, there are few reports involving experimental or feasibility studies combined with hyperthermic intrathoracic chemoperfusion therapy on cold plasma coagulation $(10,11)$. Hoffman et al (11) reported the feasibility of cold plasma coagulation using helium gas and several adverse events were reported; however, there was no reference to the prognostic impact of cold plasma coagulation. In contrast, no complications were associated with APC. Although Hoffman et al (11) combined cold plasma coagulation with hyperthermic intrathoracic chemoperfusion therapy, we did not and only performed APC intraoperatively. Although APC differs from cold plasma coagulation, the adverse events reported in the Hoffman et al study (11) might be related to a combination of hyperthermic intrathoracic chemoperfusion therapy.

The reason why our results revealed no complications related to APC might be because we exercised caution in protecting nerves, vessels, and the pericardium from the APC during radical PD. Indeed, normal organs must be protected from collateral injury during APC.

We acknowledge the limitations of this study. Our study had a retrospective single arm and consisted of a small cohort, which limited the generalizability of our results. Because this study was retrospective, we could not get patients' consents. The potential participants were given the opportunity to decline to be further enrolled in the study (opt-out). However, despite these limitations, we did not report any adverse events associated with APC and the outcomes were favorable.

We believe that APC may be able to be selected as a potential intraoperative adjuvant treatment for MPM due to the potential anti-tumor effects. We look forward to evaluating our findings in a larger and multi-center study involving APC in patients with MPM.

\section{Acknowledgements}

Not applicable.

\section{Funding}

No funding was received.

\section{Availability of data and materials}

The datasets used and/or analyzed during the present study are available from the corresponding author on reasonable request.

\section{Author's contributions}

YA, TS, SK, HO, NT, KI, AS and AI were involved in the conception and design of the study. AI wrote the manuscript. YA, TS, SK, HO and AS collected data. NT and KI supervised the study. AI and AS confirm the authenticity of all the raw data. All authors read and approved the final manuscript.

\section{Ethics approval and consent to participate}

The present study was approved by the Toho University Omori Medical Center Ethics Committee (approval no. M20085). Due to the retrospective nature of the present study, informed consent was not required from patients according to the Ethical Guidelines for Medical and Health Research involving Human Subjects of the Ministry of Health, Labour and Welfare in Japan (https://www.mhlw. go.jp/file/06-Seisakujouhou-10600000-Daijinkanboukouseikagakuka/0000080278.pdf). The present study design was accepted by the Ethics Committee on the condition that a document declaring an opt-out policy by which any possible patient and/or relatives could decline to be included in this study was uploaded on the web page of the Toho University 
Omori Medical Center (https://www.omori.med.toho-u. ac.jp/byoin/ethical_committee/pdf/rinri_M20085.pdf).

\section{Patient consent for publication}

Not applicable.

\section{Competing interests}

The authors declare that they have no competing interests.

\section{References}

1. Opitz I, Scherpereel A, Berghmans T, Psallidas I, Glatzer M, Rigau D, Astoul P, Bölükbas S, Boyd J, Coolen J, et al: ERS/ESTS/EACTS/ESTRO guidelines for the management of malignant pleural mesothelioma. Eur J Cardiothorac Surg 58: $1-24,2020$

2. Treasure T, Lang-Lazdunski L, Waller D, Bliss JM, Tan C, Entwisle J, Snee M, O'Brien M, Thomas G, Senan S, et al: Extra-pleural pneumonectomy versus no extra-pleural pneumonectomy for patients with malignant pleural mesothelioma: Clinical outcomes of the Mesothelioma and Radical Surgery (MARS) randomised feasibility study. Lancet Oncol 12: 763-772, 2011.

3. Bovolato P, Casadio C, Billè A, Ardissone F, Santambrogio L, Ratto GB, Garofalo G, Bedini AV, Garassino M, Porcu L, et al: Does surgery improve survival of patients with malignant pleural mesothelioma?: A multicenter retrospective analysis of 1365 consecutive patients. J Thorac Oncol 9: 390-396, 2014.

4. Friedberg JS, Culligan MJ, Mick R, Stevenson J, Hahn SM, Sterman D, Punekar S, Glatstein E and Cengel K: Radical pleurectomy and intraoperative photodynamic therapy for malignant pleural mesothelioma. Ann Thorac Surg 93: 1658-1665; discussion 1665-1667, 2012.
5. Bolliger CT, Sutedja TG, Strausz J and Freitag L: Therapeutic bronchoscopy with immediate effect: Laser, electrocautery, argon plasma coagulation and stents. Eur Respir J 27: 1258-1271, 2006.

6. Kesrouani A, Dabar G, Rahal S and Ghorra C: Treatment of tracheal mucoepidermoid carcinoma by argon plasma coagulation during pregnancy. Int Surg 100: 927-929, 2015.

7. Okada S, Yamauchi H, Ishimori S, Satoh S, Sugawara H and Tanaba Y: Endoscopic surgery with a flexible bronchoscope and argon plasma coagulation for tracheobronchial tumors. J Thorac Cardiovasc Surg 121: 180-182, 2001.

8. Burt BM, Richards WG, Lee HS, Bartel S, Dasilva MC, Gill RR, Jaklitsch MT, Johnson BE, Swanson SJ, Bueno R and Sugarbaker DJ: A Phase I Trial of surgical resection and intraoperative hyperthermic cisplatin and gemcitabine for pleural mesothelioma. J Thorac Oncol 13: 1400-1409, 2018.

9. Zalcman G, Mazieres J, Margery J, Greillier L, Audigier-Valette C, Moro-Sibilot D, Molinier O, Corre R, Monnet I, Gounant V, et al: Bevacizumab for newly diagnosed pleural mesothelioma in the mesothelioma avastin cisplatin pemetrexed study (MAPS): A randomised, controlled, open-label, phase 3 trial. Lancet 387: 1405-1414, 2016.

10. Hoffmann M, Ulrich A, Schloericke E, Limmer S, Habermann JK, Wolken H, Bruch HP and Kujath P: The application of cold-plasma coagulation on the visceral pleura results in a predictable depth of necrosis without fistula generation. Interact Cardiovasc Thorac Surg 14: 239-243, 2012.

11. Hoffmann M, Bruch HP, Kujath P and Limmer S: Cold-plasma coagulation in the treatment of malignant pleural mesothelioma: Results of a combined approach. Interact Cardiovasc Thorac Surg 10: 502-505, 2010.

(7) $(-)$ This work is licensed under a Creative Commons Attribution-NonCommercial-NoDerivatives 4.0 International (CC BY-NC-ND 4.0) License. 